is solvable if and only if

$$
\rho_{m}(M) \equiv \sum_{i=0}^{k-m} M^{p^{n i}} \frac{L_{m+i-1}}{L_{i}} \equiv 0 .
$$

It will be remarked that in general the first criterion is more useful. However, the latter is of some interest in itself. Furthermore, it suggests possible criteria for more general classes of congruences; I hope to develop the matter elsewhere.

DUke UnIVERSITY

\title{
A DIFFERENTIAL EQUATION FOR APPELL POLYNOMIALS $\dagger$
}

\section{BY I. M. SHEFFER}

By a set of polynomials $\left\{P_{n}(x)\right\},(n=0,1,2, \cdots)$, we shall mean an infinite sequence in which $P_{n}(x)$ is of degree exactly $\ddagger n$. Corresponding to a given set $\left\{P_{n}\right\}$ there are infinitely many sequences of polynomials $\left\{L_{n}(x)\right\}$ (with $L_{n}(x)$ of degree not exceeding $n$ ) and sequences of numbers $\left\{\lambda_{n}\right\}$ such that $\left\{P_{n}\right\}$ satisfies the linear differential equation (usually of infinite order) with parameter: $\S$

$$
L[y(x)] \equiv \sum_{n=0}^{\infty} L_{n}(x) y^{(n)}(x)=\lambda y(x),
$$

which for $\lambda=\lambda_{n}$ gives $P_{n}(x)$. In fact, suppose $\left\{P_{n}\right\}$ is given. Let $\left\{\lambda_{n}\right\}$ be any sequence of numbers subject only to the condition that $\lambda_{n}$ is not identically zero in $n$. Then a unique sequence $\left\{L_{n}(x)\right\}$ exists such that $L\left[P_{n}(x)\right]=\lambda_{n} P_{n}(x),(n=0,1, \cdots)$, where not all the $L_{n}$ 's are identically zero, and where no $L_{n}(x)$ is of degree exceeding $n$. The polynomial $L_{n}(x)$ is readily obtained by recurrence from $L_{0}, \cdots, L_{n-1}$. If we write

$$
L_{n}(x)=l_{n 0}+l_{n 1} x+\cdots+l_{n n} x^{n},
$$

$\dagger$ Presented to the Society, April 25, 1935.

$\ddagger$ For many purposes it suffices to have $P_{n}(x)$ of degree not exceeding $n$. Here, however, it is convenient to use the stricter condition.

$\S$ See Sheffer, American Journal of Mathematics, vol. 53 (1931), pp. 29-30, for a relation suggestive of (1). 
where $l_{n n}$ and other coefficients may be zero, we shall have

$$
\lambda_{n}=l_{00}+n l_{11}+n(n-1) l_{22}+\cdots+n ! l_{n n} .
$$

If we start with equation (1), that is, with the coefficients $\left\{L_{n}(x)\right\}$, and ask for what values of $\lambda$ there exists a polynomial solution, we find as a necessary condition that $\lambda$ have one of the values (3). Conversely, if $\lambda$ has any value $\lambda_{n}$ of (3), then there will correspond a polynomial solution $P_{n}(x)$ which is unique to within a multiplicative constant, provided $\lambda_{m} \neq \lambda_{n},(m \neq n)$. Moreover, $P_{n}(x)$ will be of degree exactly $n$, so that $\left\{P_{n}\right\}$ is a set. In the case in which $\lambda_{m}=\lambda_{n}$ for two (or more) distinct values of $m$ and $n$, there may or may not be, for this common characteristic number, as many linearly independent polynomial solutions as the order of the characteristic number.

Definition. Let us suppose that equation (1) is given. If for each $\lambda_{n}$ given by (3) there is a polynomial solution $P_{n}(x)$ of degree (exactly) $n$, we shall say that equation (1) is non-singular.

Corollary. Equation (1) is non-singular if $\lambda_{m} \neq \lambda_{n},(m \neq n)$.

If equation (1) is non-singular, but $\lambda_{m}=\lambda_{n}$ for some $m \neq n$, then if $P_{m}$ and $P_{n}$ are corresponding solutions of degree $m$ and $n$, the polynomial $a P_{m}+b P_{n}$ is also a solution, for every choice of $a$ and $b$. In this case the equation does not define a unique $\dagger$ set of polynomials $\left\{P_{n}\right\}$.

Because of the property of equation (1) that any set $\left\{P_{n}(x)\right\}$ is a set of solutions, (for a suitably chosen sequence $\left\{L_{n}(x)\right\}$ ), it seems appropriate to refer to (1) as a universal type equation for sets of polynomials. This comprehensive character of a type equation suggests that it may prove useful in the study of properties of sets of polynomials. We may propose the following program. Given a set of polynomials, or a class of sets; to characterize the type equations that they satisfy, and, in particular, to determine if (and when) they correspond to a type equation of finite order.

The present note investigates these questions for the class of Appell sets of polynomials.

The set $\left\{P_{n}\right\}$ is an Appell set if we have the relations

$\dagger$ We ignore the obvious arbitrary multiplicative constant attached to each $P_{n}(x)$. 


$$
\frac{d P_{n}(x)}{d x}=P_{n-1}(x), \quad(n=1,2, \cdots) .
$$

The following are well known characterizations of Appell sets.

(i) A necessary and sufficient condition that $\left\{P_{n}\right\}$ be an Appell set is that a formal power series

$$
A(t) \sim \sum_{0}^{\infty} a_{n} t^{n}
$$

exist such that

$$
e^{t x} A(t) \sim \sum_{0}^{\infty} P_{n}(x) t^{n}
$$

We shall refer to $A(t)$ as the generating function (or power series) for $\left\{P_{n}(x)\right\}$.

(ii) Set $\left\{P_{n}\right\}$ is an Appell set if and only if there exists a sequence of numbers $\left\{a_{n}\right\}$ such that $\dagger$

$$
\begin{aligned}
P_{n}(x)=a_{0} \frac{x^{n}}{n !}+a_{1} \frac{x^{n-1}}{(n-1) !}+\cdots+ & a_{n} \frac{x^{0}}{0 !}, \\
& (n=0,1, \cdots) .
\end{aligned}
$$

THEOREM 1. Let $\left\{P_{n}\right\}$ be any Appell set, with generating function $A(t)$. Define the (formal) power series

$$
B(t) \sim \sum_{0}^{\infty} b_{n} t^{n}
$$

by

$$
B(t)=\frac{A^{\prime}(t)}{A(t)} .
$$

Then $\left\{P_{n}\right\}$ satisfies the equation of type (1):

$\dagger$ In both (6) and (7), in order that $\left\{P_{n}\right\}$ be a true set, that is, for $P_{n}$ to be of degree exactly $n$, it is necessary (and sufficient) that $a_{0} \neq 0$. There is no great difficulty, however, if $a_{0}=\cdots=a_{k}=0, a_{k+1} \neq 0$. For then $P_{n}(x)$, as given by (6) and (7), is identically zero for $0 \leqq n \leqq k$, after which we get $P_{n}(x)$ of degree $n-(k+1)$; and if we define $B(t)$ by $A(t)=t^{k+1} B(t)$, then $b_{0} \neq 0$, and the Appell set for $B(t)$ is the same set as for $A(t)$ after the first $(k+1) P$ 's (which are identically zero) for $A(t)$ have been omitted. 


$$
\begin{aligned}
L\left[P_{n}(x)\right] & \equiv\left(b_{0}+x\right) P_{n}^{\prime}(x)+b_{1} P_{n}^{\prime \prime}(x)+b_{2} P_{n}^{\prime \prime \prime}(x)+\cdots \\
& =n P_{n}(x), \quad(n=0,1, \cdots) .
\end{aligned}
$$

Conversely, if the set $\left\{P_{n}\right\}$ satisfies the equation (10), where $b_{0}, b_{1}, \cdots$ are constants, then (to within multiplicative constants) $\left\{P_{n}\right\}$ is an $A$ ppell set, and its generating function $A(t)$ is defined by (9).

From (3) we see that $\lambda_{n}=n$ for equation (10). Therefore $\lambda_{m} \neq \lambda_{n},(m \neq n)$, so that to each $\lambda_{n}$ corresponds an essentially unique polynomial, and it is of degree $n$. Suppose the set $\left\{P_{n}\right\}$ satisfies (10). Let $P_{0}(x)=a_{0}$, and choose the multiplicative constants so that in $P_{n}(x)$ the coefficient of $x^{n}$ is $a_{0} / n$ !. It is verified that $P_{1}^{\prime}(x)=P_{0}(x)$. Assuming that $P_{k}^{\prime}(x)=P_{k-1}(x)$ for $k=1,2, \cdots, n-1$, we shall prove it for $k=n$. Differentiating (10), we find that $L\left[P_{n}^{\prime}(x)\right]=(n-1) P_{n}^{\prime}(x)$. Hence, since $L$ is non-singular, we have $P_{n}^{\prime}(x)=c_{n} P_{n-1}(x)$. Comparing coefficients of $x^{n-1}$, we find that $c_{n}=1$. Hence $\left\{P_{n}\right\}$ is an Appell set. Let its generating function be $A(t) \sim \sum_{0}^{\infty} a_{n} t^{n}$, where $a_{0}=P_{0}$ as already stated. Then $P_{n}(x)$ has the representation (7). If we substitute this in (10) and equate coefficients of like powers of $x$, we get the equations

$$
\begin{aligned}
b_{0} a_{n-j-1}+b_{1} a_{n-j-2}+\cdots+b_{n-j-1} a_{0} & =(n-j) a_{n-j}, \\
& (j=0,1, \cdots, n) .
\end{aligned}
$$

But these relations are completely equivalent to relation (9), as is easily verified. The converse part of Theorem 1 is thus established.

Now let $\left\{P_{n}\right\}$ be any Appell set, and let $A(t)$ be its generating function. Let us define $B(t)$ by (9), so that the coefficients of (10) are defined. Let $\left\{Q_{n}\right\}$ be the Appell set defined by (10), of whose existence (and uniqueness if we choose $Q_{0}(x)=\alpha_{0}$ ) we have just given proof. Then the generating function $A^{*}(t)$ for $\left\{Q_{n}\right\}$ is defined, by the part already proved, by the equation $B(t)=A^{* \prime}(t) / A^{*}(t)$. Since $A(0)=A^{*}(0)=a_{0}$, therefore $A^{*}(t)=A(t)$. Hence $Q_{n}(x) \equiv P_{n}(x), \quad(n=0,1, \cdots)$, so that $\left\{P_{n}\right\}$ satisfies (10). This completes the proof.

Corollary. A necessary and sufficient condition that a set $\left\{P_{n}\right\}$ be an Appell set is that it satisfy the recurrence relations 


$$
\begin{array}{r}
n P_{n}(x)=\left(b_{0}+x\right) P_{n-1}(x)+b_{1} P_{n-2}(x)+\cdots+b_{n-1} P_{0}(x), \\
(n=0,1, \cdots) .
\end{array}
$$

For if $\left\{P_{n}\right\}$ is an Appell set, it satisfies (10) and therefore (11). Now suppose $\left\{P_{n}\right\}$ is a set satisfying (11). We verify at once that $P_{1}^{\prime}=P_{0}$. Suppose $P_{k}^{\prime}=P_{k-1},(k=1,2, \cdots, n-1)$; to prove that $P_{n}^{\prime}=P_{n-1}$. If we differentiate (11) and use our induction assumption, we get $n P_{n}^{\prime}=n P_{n-1}$, so that $P_{n}^{\prime}=P_{n-1}$, and the set $\left\{P_{n}\right\}$ is an Appell set.

Relations (10) and (11) give us two further characterizations of Appell sets. But they are not the only ones possible. For example, if we want an equation (for Appell sets) of type (1) with $\lambda_{n}=n(n-1)$, then as was proved in Theorem 1 , we obtain the equation

$$
L_{[2]}\left[P_{n}(x)\right]=n(n-1) P_{n}(x),
$$

where the operator $L_{[2]}$ is defined by the equation

with

$$
L_{2}[y] \equiv \sum L_{[2] n}(x) y^{(n)}(x)
$$

$$
L_{[2]}(x ; t) \sim \sum_{n=0}^{\infty} L_{[2] n}(x) t^{n}=\frac{t^{2}}{A}\left[A^{\prime \prime}+2 x A^{\prime}+x^{2} A\right],
$$

where $A(t)$ is the generating function for $\left\{P_{n}\right\}$.

More generally, if $\left\{P_{n}\right\}$ is an Appell set defined by $A(t)$, then

$$
L_{[k]}\left[P_{n}(x)\right]=n(n-1) \cdots(n-k+1) P_{n}(x),
$$

where

$$
\begin{aligned}
L_{[k]}(x ; t) \sim & \sum_{n=0}^{\infty} L_{[k] n}(x) t^{n}=\frac{t^{k}}{A}\left[A^{(k)}+\frac{k}{1 !} x A^{(k-1)}\right. \\
& \left.+\frac{k(k-1)}{2 !} x^{2} A^{(k-2)}+\cdots+\frac{k !}{k !} x^{k} A\right] .
\end{aligned}
$$

Now suppose that we wish $\lambda_{n}$ to be a polynomial in $n$. We can suppose that $\uparrow \lambda_{0}=0$. Then we have

$$
\begin{aligned}
\lambda_{n}=n l_{11} & +n(n-1) l_{22}+\cdots \\
& +n(n-1) \cdots(n-k+1) l_{k k},
\end{aligned}
$$

$\dagger$ For this merely serves to alter the (constant) value of $L_{0}(x)$, and does not affect the polynomials $L_{n}(x)$ of index $n>0$. 
where $l_{11}, \cdots, l_{k k}$ are constants (that is, independent of $n$ ). Exactly as in Theorem 1, we obtain the following result:

THEOREM 2. Let $\left\{P_{n}\right\}$ be any Appell set and $A(t)$ its generating function. Let $\left\{\lambda_{n}\right\}$ be given by (16) for any $k \geqq 1$. Then $\left\{P_{n}\right\}$ satisfies the equation

$$
L\left[P_{n}(x)\right] \equiv \sum_{r=0}^{\infty} L_{r}(x) P_{n}^{(r)}(x)=\lambda_{n} P_{n}(x),
$$

where $L$ is defined by

$$
\begin{aligned}
L(x ; t) & \sim \sum_{r=0}^{\infty} L_{r}(x) t^{r} \\
& =l_{11} L_{[1]}(x ; t)+l_{22} L_{[2]}(x ; t)+\cdots+l_{k k} L_{[k]}(x ; t),
\end{aligned}
$$

the functions $L_{[p]}(x ; t)$ being given by (15). Conversely, given (17) with $L$ defined by (18) for any function $A(t)$, then there exists an Appell set of polynomials $\dagger\left\{P_{n}\right\}$ satisfying (17), and its generating function is precisely $A(t)$.

We proceed to characterize the equations of type (1) that define one and the same Appell set.

Theorem 3. Let $\left\{P_{n}\right\}$ be an Appell set, and $A(t)$ its generating function. $A$ necessary and sufficient condition that the set $\left\{P_{n}\right\}$ be a solution of an equation of type (1) (with $\lambda_{0}=0$, which is no restriction) is that the operator $L[y] \equiv \sum_{n=0}^{\infty} L_{n}(x) y^{(n)}(x)$ be defined $\ddagger$ by

$$
L(x ; t) \sim \sum_{n=0}^{\infty} L_{n}(x) t^{n} \sim \sum_{k=1}^{\infty} l_{k k} L_{[k]}(x ; t)
$$

where $L_{[k]}(x ; t)$ is given by (15), and the $l_{k k}$ 's are constants. The characteristic numbers $\left\{\lambda_{n}\right\}$ are given by (3).

† If $\lambda_{m} \neq \lambda_{n},(m \neq n)$, then this Appell set gives all the polynomial solutions of (17) (to within multiplicative constants). But if $m$ and $n$ (distinct) exist such that $\lambda_{m}=\lambda_{n}$, this Appell set does not include all the polynomial solutions of (17).

$\ddagger$ Although the last series in (19) may not converge, yet it serves to determine a unique sequence of polynomials $L_{1}(x), L_{2}(x), \cdots$ which are the coefficients of equation (1). For $L_{[k]}(x ; t)$, expressed as a power series in $t$, begins with a term in $t^{k}$. Hence $L_{n}(x)$, which is the coefficient of $t^{n}$ in $L(x ; t)$, is given by a finite sum, namely, the coefficient of $t^{n}$ in the sum $\sum_{k=1}^{n} l_{k k} L_{[k]}(x ; t)$. 
First we shall show that for any sequence of numbers $\left\{l_{k k}\right\}$ the Appell set $\left\{P_{n}\right\}$ satisfies equation (1) with $L$ defined by (19). Let

$$
L^{[n]}(x ; t)=\sum_{k=0}^{n} l_{k k} L_{[k]}(x ; t) \sim \sum_{r=0}^{\infty} L_{r}^{[n]}(x) t^{r},
$$

where $n$ is any positive integer. The remark of the last footnote shows that $L_{r}^{[n]}(x)=L_{r}(x),(r=0,1, \cdots, n)$. Now, by Theorem 2 , we have

$$
\begin{aligned}
L^{[n]}\left[P_{r}(x)\right] & =\left\{r l_{11}+r(r-1) l_{22}+\cdots+r ! l_{r r}\right\} P_{r}(x) \\
& =\lambda_{r} P_{r}(x), \quad(r=0,1, \cdots) .
\end{aligned}
$$

Hence

$$
L\left[P_{r}(x)\right]=L^{[n]}\left[P_{r}(x)\right]=\lambda_{r} P_{r}(x), \quad(r=0,1, \cdots, n) ;
$$

and inasmuch as $n$ can be chosen arbitrarily, it follows that $L\left[P_{r}(x)\right]=\lambda_{r} P_{r}(x)$ for all $r$. That is, $\left\{P_{n}\right\}$ satisfies equation (1). Conversely, suppose the Appell set $\left\{P_{n}\right\}$ satisfies equation (1) (with $\lambda_{0}=0$ ) ; we shall show that $L$ is defined by (19) for suitably chosen numbers $\left\{l_{k k}\right\}$. Define $\left\{l_{k k}\right\}$ by (3), and with this choice of $\left\{l_{k k}\right\}$, define an operator $L^{*}$ by (19):

$$
L^{*}(x ; t) \sim \sum_{k=1}^{\infty} l_{k k} L_{[k]}(x ; t),
$$

where the function $A(t)$ of (15) is the generating function for $\left\{P_{n}\right\}$. From the half of the theorem already established, $\left\{P_{n}\right\}$ satisfies the equation $L^{*}[y]=\lambda^{*} y$, the set $\left\{\lambda_{n}^{*}\right\}$ being given by (3). But $\left\{\lambda_{n}\right\}$ also is given by (3), so that $\lambda_{n}{ }^{*}=\lambda_{n}$. Subtracting the two equations, we find

$$
\left(L-L^{*}\right)\left[P_{n}\right]=0, \quad(n=0,1, \cdots) .
$$

If we set $n=0,1, \cdots$ successively, we find that

$$
L_{0}(x)-L_{0}^{*}(x)=L_{1}(x)-L_{1}^{*}(x)=\cdots=L_{n}(x)-L_{n}^{*}(x)=\cdots=0 .
$$

That is, $L^{*} \equiv L$. This proves the converse.

We next determine the condition that an Appell set satisfy a finite order equation of type (1). Two well known examples are the polynomial sets $\left\{(x-a)^{n} / n !\right\}$ and $\left\{H_{n}(x)\right\}$, the latter being 
the Hermite polynomials. They satisfy the following equations, respectively:

$$
\begin{aligned}
& L[y(x)] \equiv(x-a) y^{\prime}(x)=\lambda y(x), \quad\left(\lambda_{n}=n\right) ; \\
& L[y(x)] \equiv 2 x y^{\prime}(x)-y^{\prime \prime}(x)=\lambda y(x), \quad\left(\lambda_{n}=2 n\right) .
\end{aligned}
$$

The corresponding generating functions are respectively $A(t)=e^{-\alpha t}$ and $A(t)=e^{-t^{2} / 4}$.

Theorem 4. A necessary and sufficient condition that an Appell set $\left\{P_{n}\right\}$, with generating function $A(t)$, satisfy a finite order equation of type (1) is that $A(t)$ have the form

$$
A(t)=e^{Q(t)},
$$

where $Q(t)$ is a polynomial.

Suppose that $A(t)$ has the form (20), where $Q$ is of degree $k$. Then from (9), $B(t)=Q^{\prime}(t)$, a polynomial of degree $k-1$, so that $B(t)=b_{0}+b_{1} t+\cdots+b_{k-1} t^{k-1}$. The universal equation (10) for Appell polynomial sets therefore reduces to

$$
\begin{aligned}
& L\left[P_{n}(x)\right]=\left(b_{0}+x\right) P_{n}^{\prime}(x)+b_{1} P_{n}^{\prime \prime}(x)+\cdots+b_{k-1} P_{n}^{(k)}(x) \\
& =n P_{n}(x) \text {, } \\
& (n=0,1, \cdots) \text {, }
\end{aligned}
$$

and this is an equation of finite order (namely, order $k$ ).

Conversely, suppose the Appell set $\left\{P_{n}\right\}$ satisfies a finite order equation of type (1); we shall show that its generating function $A(t)$ has the form (20). Let the equation be

$$
\begin{array}{r}
L\left[P_{n}(x)\right] \equiv L_{0}(x) P_{n}(x)+\cdots+L_{k}(x) P_{n}^{(k)}(x)=\lambda_{n} P_{n}(x), \\
(n=0,1, \cdots) .
\end{array}
$$

As already pointed out, we may suppose that $\lambda_{0}=0$ (so that $\left.L_{0}(x)=0\right)$. If we write $L_{n}(x)$ in the form (2), then $\lambda_{n}$ is given by (3), but since $L_{n}(x) \equiv 0,(n>k), \lambda_{n}$ reduces to the form (16). Let $L_{[k]}(x ; t),(k=1,2, \cdots)$, be the functions of $(15)$, and define the operator $L^{*}$ by $L^{*}(x ; t)=\sum_{r=1}^{k} l_{r r} L_{[r]}(x ; t)$, where $A(t)$ is the generating function for $\left\{P_{n}\right\}$. Then $\left\{P_{n}\right\}$ satisfies (by Theorem 2) the equation $L^{*}\left[P_{n}\right]=\lambda_{n}^{*} P_{n}$, where $\lambda_{n}^{*}$ is determined by (16). Since (16) was used to determine $\lambda_{n}$, it follows 
that $\lambda_{n}{ }^{*}=\lambda_{n}$ for all $n$. Hence (as was argued before) we have $L^{*} \equiv L$. That is, the given equation for $\left\{P_{n}\right\}$ is defined by

$$
L(x ; t)=\sum_{r=1}^{k} l_{r r} L_{[r]}(x ; t) .
$$

But to begin with we had

$$
L(x ; t)=\sum_{r=1}^{k} L_{r}(x) t^{r}
$$

Hence

$$
\sum_{r=1}^{k} l_{r r} L_{[r]}(x ; t)=\sum_{r=1}^{k} L_{r}(x) t^{r}
$$

Let

(d) $\quad \frac{A^{(r)}(t)}{A(t)}=a_{r 0}+a_{r 1} t+\cdots, \quad(r=0,1, \cdots, k)$.

Expanding the left member of (c) in a power series in $t$, and observing (from the right member) that powers of $t$ higher than the $k$ th degree cannot occur, we obtain the equations

$$
\begin{aligned}
l_{11}\left(a_{1 n}\right) & +l_{22}\left(a_{2, n-1}+2 x a_{1, n-1}\right)+\cdots \\
& +l_{k k}\left(a_{k, n-k+1}+\frac{k}{1 !} x a_{k-1, n-k+1}+\cdots\right. \\
& \left.+\frac{k(k-1) \cdots 2}{(k-1) !} x^{k-1} a_{1, n-k+1}\right)=0, \quad(n \geqq k) .
\end{aligned}
$$

These relations are to hold identically in $x$. Of the numbers $1, \cdots, k$, let $s$ be the largest for which $l_{s s} \neq 0$. It then follows from (e) that

$$
a_{1, n-8+1}=0, \quad(n \geqq k) .
$$

That is,

$$
\frac{A^{\prime}(t)}{A(t)}=B(t)=a_{10}+a_{11} t+\cdots+a_{1, k-8} t^{k-8},
$$


so that (20) holds, where $Q(t)$ is a polynomial of degree $k-s+1$. This completes the proof.

If the Appell set $\left\{P_{n}\right\}$ satisfies an equation of type (1) of finite order, then there are infinitely many other equations (of type (1)) of finite order for which the set is a solution. For example, from $L\left[P_{n}\right]=\lambda_{n} P_{n}$ follows $L^{2}\left[P_{n}\right]=\lambda_{n}{ }^{2} P_{n}, L^{3}\left[P_{n}\right]=\lambda_{n}^{3} P_{n}$, . . . This raises the question: What is the minimum order of all equations satisfied by $\left\{P_{n}\right\}$ ? The answer is given by the following statement.

Theorem 5. Let the Appell set $\left\{P_{n}\right\}$ satisfy a finite order equation of type (1), so that its generating function $A(t)$ is given by (20). Then the minimum order of all equations (of type (1)) satisfied by $\left\{P_{n}\right\}$ is precisely the degree $\dagger$ of the polynomial $Q(t)$.

Let $Q(t)$ be of degree $m$. That the minimum order $k$ cannot exceed $m$ is shown by the proof of the first half of Theorem 4 , so that $k \leqq m$. Let $\ddagger L\left[P_{n}\right]=\lambda_{n} P_{n}$ be the equation of minimum order $k$. From the proof of the second part of Theorem 4 , we see that there is an integer $s$ for which $1 \leqq s \leqq k$, and such that $Q(t)$ is of degree $k-s+1$. That is, $m=k-s+1$. But $k \leqq m$, so that $k \leqq k-s+1$. This requires that $s=1$, from which follows the desired conclusion that $k=m$.

Pennsylvania State College

$\dagger$ The single exception is when $Q(t)$ is of degree zero, in which case $A(t)$ is a constant and $P_{n}(x)=c x^{n} / n$ !. This set satisfies a first order equation.

$\ddagger$ As usual it is no restriction to suppose, as we do, that $\lambda_{0}=0$. 